\title{
SUPPRESSION OF THE DAYSIDE MAGNETOPAUSE SURFACE MODES
}

\author{
V.A. Pilipenko \\ Institute of Physics of the Earth, \\ Moscow, Russia, pilipenko_va@mail.ru \\ O.V. Kozyreva \\ Institute of Physics of the Earth, \\ Moscow, Russia, kozyreva@ifz.ru \\ L. Baddeley \\ University Centre in Svalbard \\ Svalbard, Norway \\ Birkeland Center for Space Science \\ Birkeland, Norway, lisa.baddeley@unis.no
}

\author{
D.A. Lorentzen \\ University Centre in Svalbard \\ Svalbard, Norway, \\ Birkeland Center for Space Science \\ Birkeland, Norway, dag.lorentzen@unis.no \\ V.B. Belakhovsky \\ Polar Geophysical Institute, \\ Apatity, Russia,belakhovsky@mail.ru
}

\begin{abstract}
Magnetopause surface eigenmodes were suggested as a potential source of dayside high-latitude broadband pulsations in the Pc5-6 band (frequency about 1-2 mHz). However, the search for a ground signature of these modes has not provided encouraging results. The comparison of multi-instrument data from Svalbard with the latitudinal structure of Pc5-6 pulsations, recorded by magnetometers covering near-cusp latitudes, has shown that often the latitudinal maximum of pulsation power occurs about $2-3^{\circ}$ deeper in the magnetosphere than the dayside open-closed field line boundary (OCB). The OCB proxy was determined from SuperDARN radar data as the equatorward boundary of enhanced width of a return radio signal. The OCB-ULF correspondence is further examined by comparing the latitudinal profile of the near-noon pulsation power with the equatorward edge of the auroral red emission from the meridian scanning photometer. In most analyzed events, the "epicenter" of Pc5-6 power is at $1-2^{\circ}$ lower latitude than the optical OCB proxy. Therefore, the dayside Pc5-6 pulsations cannot be associated with the ground image of the magnetopause surface modes or with oscillations of the last field line. A lack of ground response to these modes beneath the ionospheric projection of OCB seems puzzling. As a possible explanation, we suggest that a high variability of the outer magnetosphere near the magnetopause region may suppress the
\end{abstract}

excitation efficiency. To quantify this hypothesis, we consider a driven field line resonator terminated by conjugate ionospheres with stochastic fluctuations of its eigenfrequency. A solution of this problem predicts a substantial deterioration of resonant properties of MHD resonator even under a relatively low level of background fluctuations. This effect may explain why there is no ground response to magnetopause surface modes or oscillations of the last field line at the OCB latitude, but it can be seen at somewhat lower latitudes with more regular and stable magnetic and plasma structure.

Keywords: ULF waves, magnetopause, open-closed boundary, MHD surface modes, Alfven resonator.

\section{IONOSPHERIC FOOTPRINT OF THE DAYSIDE OCB FROM OPTICAL AND HF RADAR OBSERVATIONS}

The open-closed field line boundary (OCB) is the boundary that separates magnetospheric field lines that are dragged by the interplanetary magnetic field from those that are closed within the magnetosphere. The ability to monitor the OCB location allows us to study the electrodynamics of magnetosphere-ionosphere coupling, in particular to estimate energy storage and release in the magnetosphere. There are several methods for discriminating the ionospheric footprint of the dayside OCB, but each of them has some merits and drawbacks.

The open/closed separatrix can be identified using particle precipitation boundaries observed by lowaltitude spacecraft [Newell, Meng, 1988; Oksavik et al., 2000]. The poleward edge of high-energy (>10 keV) electron precipitation ('trapping boundary") corresponds to particles trapped on closed field lines. Field lines poleward of this region must therefore be open. However, the measurements can be made with cadence about 90 min and along orbit only.

Precipitating soft electrons produce plasma irregularities, and strong coherent backscatter from these irregularities can be monitored by Super Dual Auroral Radar Network (SuperDARN) radars. The spectral width of the Doppler velocity of measured backscattered signal can be interpreted as a manifestation of plasma turbulence of the scattering region. The transition from narrow to broad spectral widths has been utilized to investigate the location of ionospheric bounda- 
ries [Baker et al., 1995]. Many studies have revealed that the high-latitude broad Doppler spectral width can be used as an indicator for the cusp region [Moen et al., 2001], even though the interpretation of the broad spectral width is still elusive. However, ionospheric regions with elevated turbulence level can be associated not only with the cusp proper, but with the low-latitude boundary layer; hence, this method may provide ambiguous results.

A demarcation of the cusp soft electron precipitation can be determined by comparing the equatorward boundary of the red $(630.0 \mathrm{~nm})$ auroral emission along the photometer meridian scanning [Lorentzen et al., 1996]. Precipitation of soft electrons with $0.1-1 \mathrm{keV}$ energies is a good indicator of open magnetic field lines and corresponds to the cusp aurora dominated by the red emission (Rayleigh intensity ratio 630.0/557.7>1) [Lorentzen, Moen, 2000; Johnsen, Lorentzen, 2012]. However, dayside auroral optical measurements can be made during winter months only.

The co-location of the equatorward edge of the HF radar cusp and the cusp auroral emission has been extensively studied [Yeoman et al., 1997]. Rodger et al. [1995] showed that the radar cusp signatures were located $\sim 0.5^{\circ}$ equatorward of the optical cusp, whereas Milan et al. [1999] found that the equatorward boundaries of the optical cusp were, on average, located at slightly lower latitudes than the radar cusp. Comparing the spectral width method to the optical method, the radar OCB proxy was seen to be on average $0.5-1.6^{\circ}$ (56-170 $\mathrm{km})$ poleward of the optical OCB [Chen et al., 2015].

A new discriminator of OCB was suggested on the basis of ground magnetometer observations of highlatitude ultra-low-frequency (ULF) activity. The background of this tool is described in the following section.

\section{HIGH-LATITUDE ULF WAVE ACTIVITY AS A POSSIBLE OCB DISCRIMINATOR}

Geomagnetic pulsations in the Pc5-6 band (periods about 3-15 min) have been known to be a persistent feature of the ULF activity at dayside high latitudes [Engebretson et al., 1995; Francia et al., 2005]. Morphologically, these high-latitude long-period pulsations (sometimes called Irregular Pulsations at Cusp Latitudes [Bolshakova et al., 1988]) differ from well-known quasi-monochromatic Pc5 waves observed at auroral latitudes [Kozyreva et al., 2016]. The specific high-latitude near-noon pulsations could be a signature of the magnetopause surface eigenmodes [Plaschke et al., 2009a; Hartinger et al., 2015] or oscillations of the last field lines [Lanzerotti et al., 1999; Urban et al., 2011]. Away from the near-noon sector, at dawn and dusk flanks of the magnetopause, surface modes are strongly coupled with the Kelvin-Helmholtz instability of the magnetosheath plasma flow around the magnetosphere [Mazur, Chuiko, 2013]. Ultra-low "magic" geomagnetic pulsation frequencies were reinterpreted in terms of standing Alfvén surface mode of discrete eigenfrequencies (or Kruskal-Schwarzschild modes) at the magnetopause [Plaschke et al., 2009b]. Away from the subsolar magnetopause, it is unlikely that there are standing surface waves [Plaschke, Glassmeier, 2011]. Observations of the magnetospheric response to jets in the subsolar magnetosheath showed that though broadband jets do not exhibit discrete frequencies, they do drive waves at the discrete "magic" frequencies [Archer et al., 2013]. Statistical evidence for power enhancements in the daytime spectra at the frequencies close to expected magic frequencies was found at a very high latitude station in Antarctica [Villante et al., 1997], though larger statistical studies revealed little or no spectral peaks [Plaschke et al., 2008]. The magnetopause surface mode eigenfrequency, about $1 \mathrm{mHz}$, was estimated by oversimplified models [Archer, Plaschke, 2015], so it should be treated with caution.

Narrowband (1-4 mHz) Pc 5 activity, interpreted as Alfvén oscillations of the outermost closed field lines, was also suggested to differentiate between open and closed field line regions near local noon [McHarg et al., 1995; Lanzerotti et al., 1999]. The feasibility to monitor the OCB structure through the use of ULF observations at the Antarctic network of magnetometers was proven by Urban et al. [2011]. OCB has been identified and mapped by analyzing Pc5 ( 3-9 min) and Pc6 ( 10-20 min) oscillations. The presence/absence of these particular periodicities was assumed to be an indicator of closed/open field lines, and thus it allows us to distinguish OCB.

A ground response to the magnetopause surface modes should be expected beneath the ionospheric projection of OCB. The association of dayside high-latitude ULF activity with OCB has been unambiguously resolved using the Svalbard observational complex. It comprises the latitudinal IMAGE magnetometer chain along geomagnetic longitude $\Lambda \sim 110^{\circ}$ (MLT noon is $\sim 09$ UT), SuperDARN radar facility, and meridian scanning photometer. Svalbard is very well situated for dayside cusp auroral observations. The Finland SuperDARN radar overlaps the field of view of the optical instruments. These high-latitude ground-based instruments offer a unique opportunity to monitor the motion of the polar cap boundary in near real-time. Here we present two typical examples of the comparison between the latitudinal structure of broadband Pc5-6 pulsations, recorded by magnetometers covering near-cusp latitudes, and the dayside OCB proxy, interpreted either as an enhanced spectral width of the SuperDARN radar return signal or as the equatorward boundary of the cusp aurora determined by the scanning photometer. The obtained result imposes an important limitation on possible mechanisms of high-latitude ULF variations.

\section{DAYSIDE OCB FROM HF RADAR AND ULF ACTIVITY}

The Cooperative UK Twin Located Auroral Sounding System (CUTLASS) radar, which is one of the SuperDARN radars, is located at Hankasalmi, Finland. The 16 phased antenna arrays, operating at frequencies between 8 and $20 \mathrm{MHz}$, cover $52^{\circ}$ in the azimuthal direction. We present data from the normal scan mode employing a dwell time of $7 \mathrm{~s}$ at each beam for a full 2 min scan. 
We give a typical example of the observed correspondence between dayside ULF power structure and OCB proxy, determined from SuperDARN radar data. The SuperDARN data on the 2002 January 04 event, containing the ionospheric velocity pattern and the spectral width of radio signal returns, indicate that the cusp equatorward boundary from $\sim 07: 30$ UT to $\sim 11: 00$ UT was on average around a geographic latitude of $76^{\circ}$ (Figure 1, upper panel).

Along the latitudinal magnetometer array, quasiperiodic variations with time scales from $\sim 7 \mathrm{~min}$ to $\sim 15$ min are observed with peak-to-peak amplitudes of $>10$ nT (Figure 1, middle panel). These variations are most evident in the X-component from $\sim 07$ UT to 10 UT at stations Hopen Island (HOP) at the geomagnetic latitude $\Phi=72.9^{\circ}$ and Bear Island (BJN) at $\Phi=71.4^{\circ}$.

Fourier spectra demonstrate a dominance of $\sim 0.6$ and $\sim 1.5 \mathrm{mHz}$ frequencies (not shown). The latitudinal distribution of X-component spectral power for the time period 07-10 UT integrated in the $0.4-3.0 \mathrm{mHz}$ band shows a maximum around $73-74^{\circ}$ (Figure 1 , bottom panel). Thus, the ULF maximum is shifted $\sim 2^{\circ}$ equatorward from the cusp boundary (vertical dashed line). Other events and larger statistics confirming characteristic features presented in this example are discussed in [Pilipenko et al., 2015].

\section{DAYSIDE OPTICAL OCB AND ULF ACTIVITY}

To verify the results obtained with the SuperDARN radar, we examine a typical event, where the meridian scanning photometer is used to identify the cusp aurora equatorward boundary (dayside OCB proxy). This instrument, located at Longyearbyen (LYR) at $\Phi \sim 75.3^{\circ}$, provides optical observations with $16 \mathrm{~s}$ temporal and $1^{\circ}$ angular resolution. There are five channels, including the $630.0 \mathrm{~nm}$ red and $557.7 \mathrm{~nm}$ green auroral emissions. The photometer has the highest spatial resolution near the zenith, where auroral height ambiguities are negligible. Beam 9 of the Finland SuperDARN radar intersects with the LYR photometer at the $\sim 1890 \mathrm{~km}$ ground range. However, simultaneous SuperDARN and LYR photometer observations are very rare [Chen et al., 2015].

We show the correspondence between the optical proxy of OCB and the latitudinal structure of wave power in the Pc5-6 band as inferred from magnetometer data.

We examine the 1994 December 30 event when dynamic auroral activity was observed over the LYR station. We concentrate on the near-noon time interval, 09-10 UT. During this interval, quasi-periodic irregular pulsations were recorded along the IMAGE magnetometer chain. The spectra of magnetic variations during this time interval revealed two dominating spectral peaks: at $\sim 1.4 \mathrm{mHz}$ and $\sim 2.2 \mathrm{mHz}$ in both $\mathrm{X}$ and $\mathrm{Z}$ components (not shown).

A magnetic "keogram", constructed from the Xcomponent magnetometer data (Figure 2), enables us to monitor the time evolution of the latitudinal distribution of band-integrated spectral power along the geomagnetic meridian. The superposition of the magnetic keogram of ULF power in the $1.7-2.0 \mathrm{mHz}$ band with the equatorward and poleward boundaries of the cusp aurora (red and blue lines respectively) indicates that the Pc6 pulsation power is located $\sim 1-2^{\circ}$ equatorward of the optical OCB. We have found many other events with a similar auroral OCB - ULF power. The dedicated statistical study confirming the above result is under way.

The presented examples illustrate a commonly observed regularity: a ground response to driving of the outer magnetosphere occurs not beneath the last closed field line, but somewhat deeper in the magnetosphere. The latitudinal maximum of the broadband near-noon Pc6 pulsations takes place very close to OCB, but somewhat deeper inside the magnetosphere.

To interpret the observed correspondence between dayside ULF power structure and OCB proxy, determined either from SuperDARN or auroral optical data, we use a simplified model of MHD resonator with fluctuating eigenfrequency driven by an external source.

\section{THEORETICAL BACKGROUND OF ALFVÉN AND SURFACE MODES}

The basic theoretical notions on MHD oscillations in the magnetosphere were originally derived from the simplified "plasma box" model with 1D inhomogeneity. More complicated geometries considered confirmed the fundamental results of the "plasma box" model [Nakarjakov et al., 2016]. A characteristic frequency $\Omega_{\mathrm{A}}$ of Alfvén field line oscillations is mainly determined by the region near the top of a field line with the Alfvén velocity minimum. In a dipole-like magnetic field, it can be estimated as [Yumoto et al., 1995]

$$
\Omega_{A}=\frac{V_{A}}{L R_{E}},
$$

where $V_{A}$ is the Alfvén velocity near the top of a field line, and $\mathrm{LR}_{\mathrm{E}}$ is the radial distance to the top of a field line. The Alfvén wave continuum is terminated by the frequency of the last closed field line.

Properties of MHD surface mode are similar to that of Alfvén waves: both are non-compressional disturbances and are guided along the background magnetic field B. Both Alfvén field line oscillations and surface waves on a steep gradient of plasma parameters are modes of the field-aligned MHD resonator terminated by the conjugate ionospheres. The frequency of a surface mode $\Omega_{\mathrm{S}}$ lies between the Alfvén frequencies at both sides $\left(\Omega_{A}^{1}, \Omega_{A}^{2}\right)$ of the interface between two media (e.g., magnetosphere and magnetosheath with magnetic field $B_{1,2}$ and plasma density $\rho_{1,2}$ ) [Chen, Hasegawa, 1974]

$$
\Omega_{S}=\frac{1}{L R_{E}} \sqrt{\frac{B_{1}^{2}+B_{2}^{2}}{\mu\left(\rho_{1}+\rho_{2}\right)}} \quad \Omega_{A}^{1}<\Omega_{S}<\Omega_{A}^{2}
$$

The surface mode amplitude exponentially decays from the boundary with the scale length $\propto\left|k_{\|}\right|^{-1}$, which for the first three harmonics vary from $\sim 8 R_{\mathrm{E}}$ to $\sim 2.8 R_{\mathrm{E}}$ [Archer, Plascke, 2015]. Thus, these harmonics could potentially be recorded even at the geostationary orbit. A dominant damping mechanism determines the width of a ULF resonant peak and the effective Q-factor of the magnetospheric resonator. Commonly, Joule dissipation in the ionosphere is considered as a prevailing damping mechanism at middle and low latitudes [Newton et al., 1978] determined by the height-integrated 

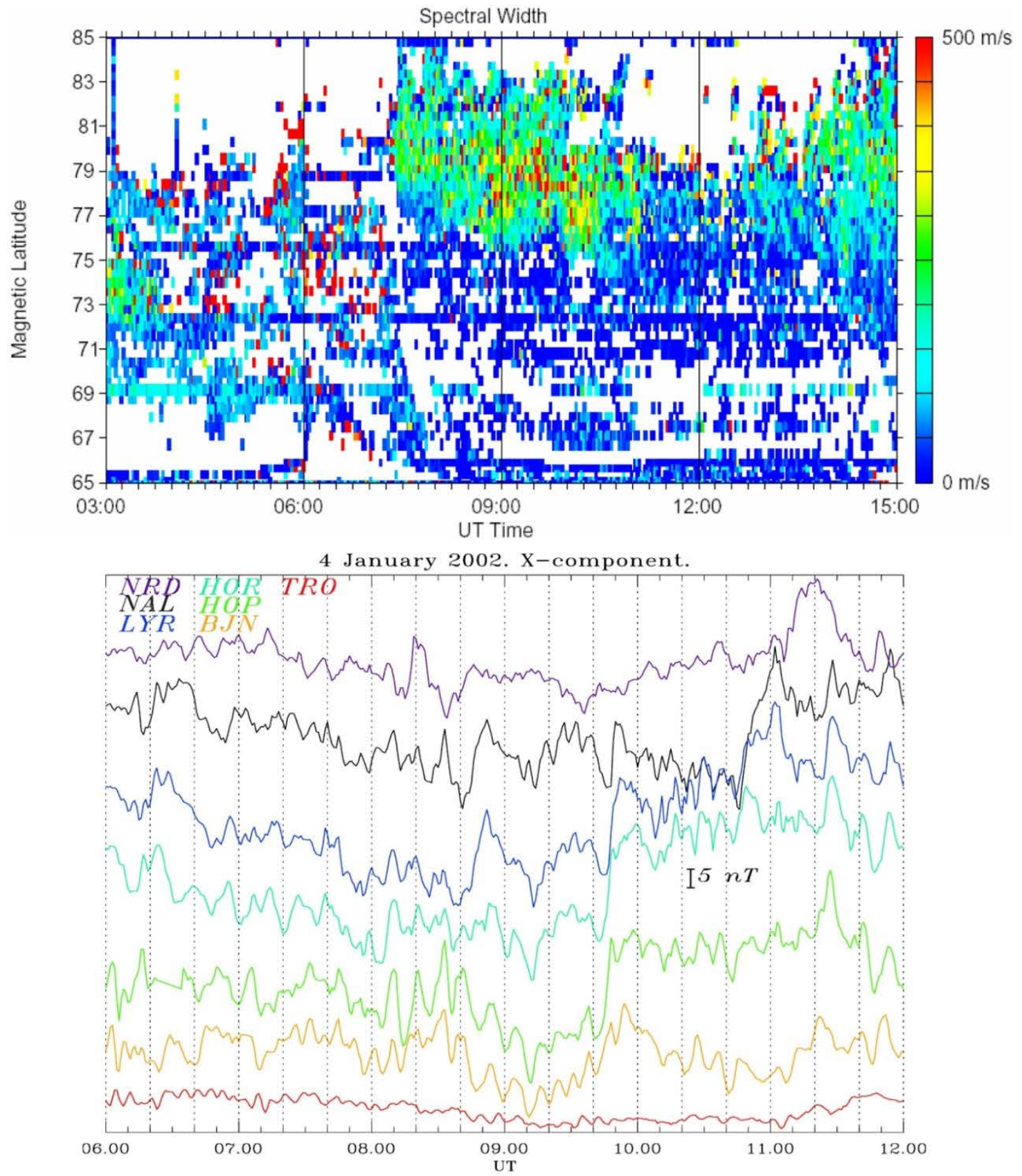

4 January 2002. 0.4-3 mHz. 07.00-10.00 UT.

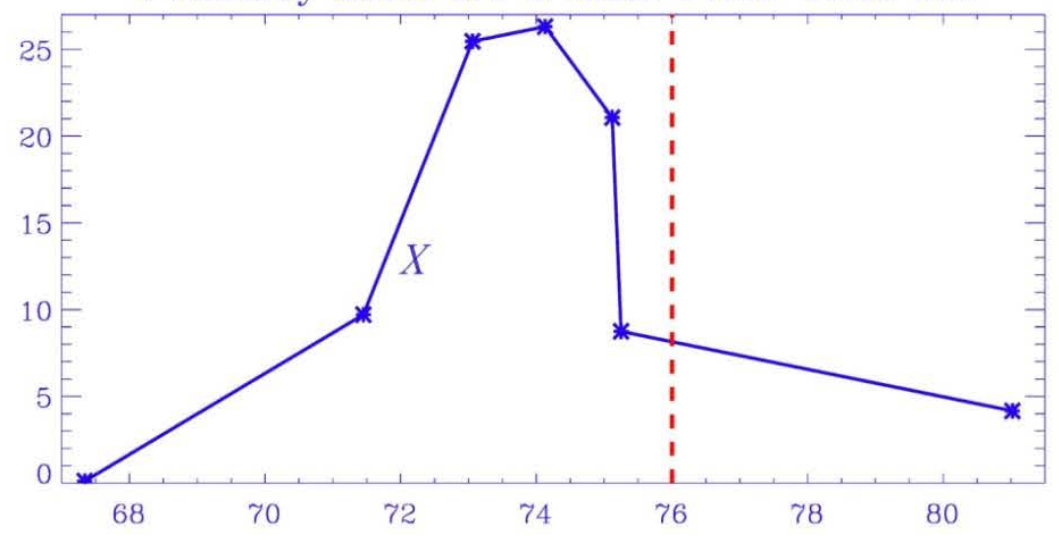

Figure 1. The 2002 January 04 event: spectral width of SuperDARN radio signal returns (upper panel); geomagnetic variations (X-component) along the IMAGE magnetometer chain (middle panel); the latitudinal distribution of X-component spectral power for the time period 07:00-10:00 UT integrated in the $0.4-3.0 \mathrm{mHz}$ band (bottom panel). The equatorward cusp boundary is shown by the vertical dashed line 
Pedersen conductance. Additional dissipative mechanisms can operate at auroral latitudes such as field-aligned potential drop [Fedorov et al., 2001], anomalous plasma resistance [Lysak, Dum, 1983], transformation into smallscale dispersive modes [Leonovich, Mazur, 1989], and non-linear saturation [Rankin et al., 1999].

In a realistic inhomogeneous magnetosphere, global MHD disturbances (e.g., surface modes) are to be inevitably coupled to local standing field line Alfvén oscillations, most effectively in a resonant region, where the local Alfvén period matches a periodicity in a surface mode of the wideband spectrum. According to the MHD spectral theory, the surface mode does not constitute an eigenoscillation of any inhomogeneous plasma system. Even without dissipation in a system, the surface mode with discrete spectrum irreversibly converts into a continuum of Alfvén oscillations and can exist for a limited time, decaying with rate $\gamma / \omega \sim(\mathrm{ka})$, where a is the width of an interface, and $\mathrm{k}$ is the transverse wave number [Chen, Hasegawa, 1974]. Therefore, only short-lived transient Pc6 oscillations can be associated with possible magnetopause modes, whereas long-lasting quasiperiodic oscillations are probably related to the resonant response of inner magnetospheric shells, at lower latitudes. Moreover, there is another factor, considered below, which may suppress the surface mode excitation by an external driver owing to system background fluctuations.

\section{DRIVING RESONATOR WITH STATIONARY BACKGROUND FLUCTUATIONS}

In attempt to comprehend the presented observational results, we take into account that magnetospheric plasma and background magnetic field experience substantial stochastic fluctuations, especially in the outer magnetosphere near the magnetopause. Hence, the eigenfrequency $\Omega$ should also experience stochastic fluc- tuations. We consider a simple model of homogeneous MHD resonator terminated by the conjugate ionospheres with stochastic eigenfrequency fluctuation. This model enables us to examine possible suppression and deterioration of field line resonant response to an external driving.

We consider a simple model of homogeneous resonator terminated by conjugated ionospheres. Let us suppose that its eigenfrequency $\Omega$ experiences stationary stochastic fluctuations as follows

$$
\Omega^{2}(t)=\Omega^{2}[1+\delta \xi(t)],
$$

where $\xi(t)$ is the stationary stochastic function with vanishing time-average $\left\langle\xi>=0\right.$ and unit dispersion $\left\langle\xi^{2}\right\rangle=1$. The relevant auto-correlation function $K(\tau)$ satisfies the condition $\left\langle\xi^{2}\right\rangle=K(0)=1$. The parameter $\delta$ characterizes the amplitude of eigenfrequency fluctuations such that $\left|\Delta \Omega^{2} / \Omega^{2}\right| \sim \delta$.

The equation for field line oscillations driven by largescale compressional waves or for surface waves buffeted by the magnetosheath disturbances is formally reduced to the equation for a driven harmonic oscillator with eigenfrequency $\Omega$ [Hollweg, 1997]. This modeling equation describes oscillations characterized by a variable $x(t)$ (magnetic or electric field, plasma velocity or displacement, etc.) as follows

$$
x_{t t}+2 \gamma x_{t}+\Omega^{2}[1+\delta \xi(t)] x=A \Omega^{2} \cos \omega t .
$$

Here $\mathrm{A}$ is the driver amplitude, and $\gamma$ is the damping factor of the resonator.

In the case of low level background fluctuations such that $\delta<<1$, the approximate solution of (1) can be found by the iteration method, developed in the theory of nonlinear stochastic mechanics [Dimentberg, 1980; Gardiner, 2004]. Beyond the small- $\delta$ approximation, this equation has been numerically solved in [Coult et al., 2007]. We seek a solution in the following form:

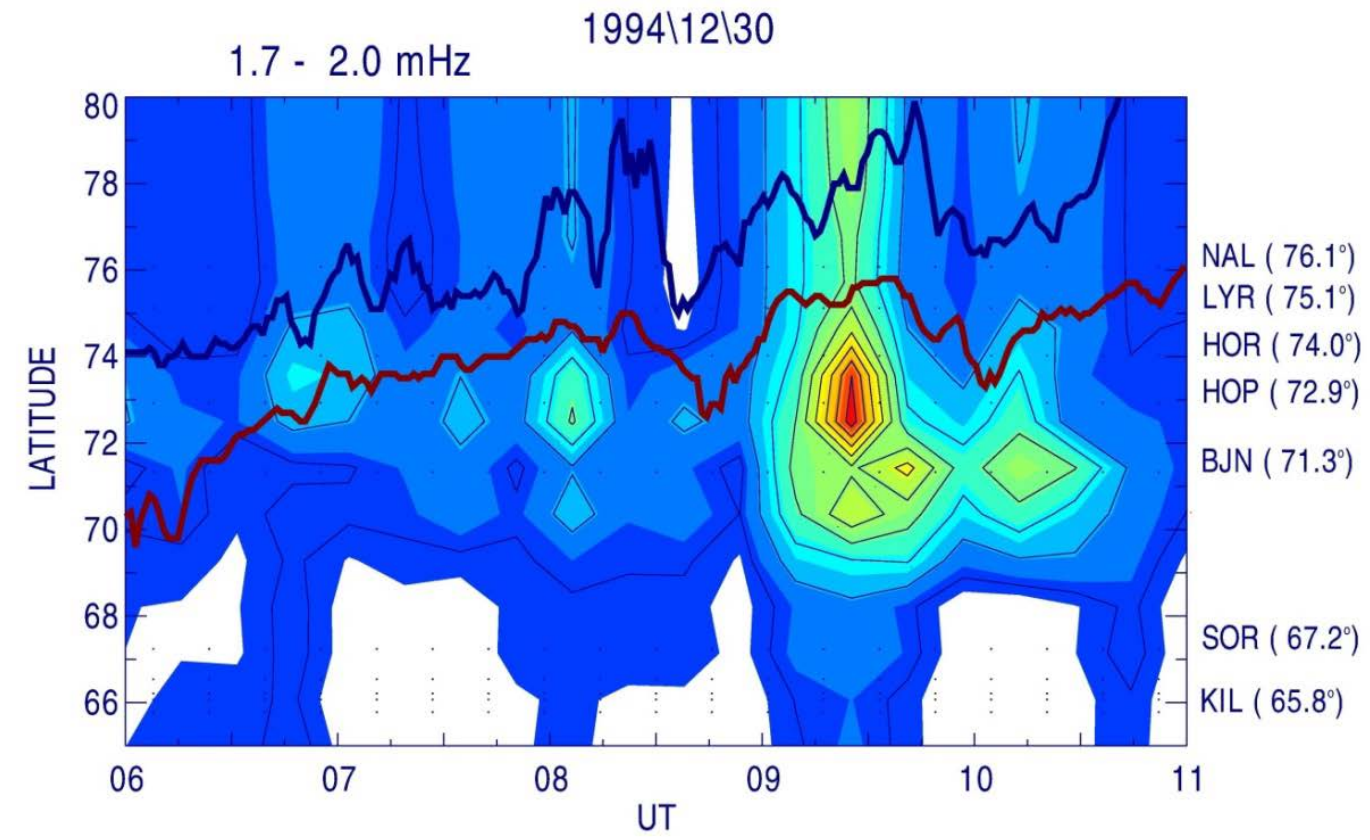

Figure 2. The 1994 December 30 event: magnetic keogram constructed from the X-component IMAGE magnetometer data and superposed onto the equatorward and poleward boundaries of the cusp aurora (red and blue lines respectively) 


$$
x(t)=x_{0}(t)+\delta x_{1}(t)+\delta^{2} x_{2}(t)+\ldots
$$

Substituting (2) into (1) and grouping all the terms of the same $\delta$ order provides an infinite system of equations in respect to $x_{i}(t)$ (see Appendix for details). In the 0 -approximation, we can obtain the classical formula for a driven linear oscillator

$$
\begin{aligned}
& x_{0}(t)=b_{0} \sin \left(\omega t+\varphi_{0}\right), \\
& b_{0}=\frac{A \Omega^{2}}{\sqrt{\left(\Omega^{2}-\omega^{2}\right)^{2}+4 \gamma^{2} \omega^{2}}}, \\
& \varphi_{0}=\arctan \frac{\Omega^{2}-\omega^{2}}{2 \gamma \omega} .
\end{aligned}
$$

Features of this solution are the occurrence of a resonant peak and a phase reversal near the resonance $\omega \rightarrow \Omega$. In the absence of background fluctuations, the peak amplitude $b_{0}^{(\max )}$ and the semi-width of the spectral peak $\Delta \omega$ are determined by the damping factor $\gamma$ of the system, or otherwise by the $Q$-factor $Q=\Omega / 2 \gamma$, as follows

$$
\frac{b_{0}^{(\max )}}{A}=Q ; \quad \frac{\Delta \omega}{\Omega}=\frac{1}{2 Q} .
$$

In subsequent approximations $(\mathrm{i}=1,2, \ldots)$, the fluctuation-induced correction to the solution can be found from recurrence formula (A1) as shown in the Appendix. Keeping terms up to $\delta^{2}$ order, from the relationships (A2) and (A3) it follows

$$
\begin{aligned}
& \langle x(t)\rangle=b \sin \left(\omega t+\varphi_{0}-\varphi_{1}\right), \\
& \frac{b(\omega)}{b_{0}}=1-\delta^{2} \times \\
& \times \frac{\Omega^{4}\left\{2 \gamma \omega\left[J^{(-)}-J^{(+)}\right]+\left(\Omega^{2}-\omega^{2}\right)\left[I^{(-)}-I^{(+)}\right]\right\}}{2 \omega_{1}^{2}\left[\left(\Omega^{2}-\omega^{2}\right)^{2}+4 \gamma^{2} \omega^{2}\right]},
\end{aligned}
$$

where $\omega_{1}^{2}=\Omega^{2}-\gamma^{2}$. The additional phase shift $\varphi_{1} \simeq o\left(\delta^{2}\right)$ in (5) is of no importance to us. The relationship (5) predicts that the amplitude of the resonant oscillations should change under the influence of stochastic fluctuations of eigenfrequency. Near the resonance, as $\omega \rightarrow \Omega$, it follows from (5) that

$$
\frac{b}{b_{0}^{(\max )}} \simeq 1-\delta^{2} \frac{\Omega^{3}}{4 \gamma \omega_{1}^{2}}\left[J^{(-)}-J^{(+)}\right] .
$$

As shown in the Appendix, from (6) it follows that when the spectral density $\Phi(\omega)$ of $\xi(t)$ fluctuations is a non-growing function, these fluctuations cause a decrease in the average amplitude of resonant oscillations. The considered deterioration of resonator properties is not related to the occurrence of anomalous resistivity or viscosity in a turbulent plasma as in [Hollweg, 1987; Borovsky et al., 1997]. In fact, a decrease in the resonant response to an external driving is caused by stochastic deviations of eigenfrequency from exact resonance and mismatch between the driver frequency and resonator eigenfrequency. Thus, this mechanism is a non-dissipative one, i.e. it does not introduce any additional energy absorption into a system.
We demonstrate how the system response varies with the amplitude of fluctuations characterized by $\delta$. Several examples of the influence of the background fluctuations on the frequency response amplitude are shown in Figure 3. In this example, we use the fluctuations with power spectrum $1 /$ f. Without fluctuations, the classic curve shows a sharp resonant peak near $\Omega=\omega$. The addition of fluctuations reduces the height of the resonant peak and increases its width, whereas the effect is more pronounced for a larger $\delta$. The height of the peak is determined by the effective $Q$ factor of the system.

The suppression of resonant oscillation depends essentially on the spectrum of fluctuations. For a model problem, we choose noise functions defined by fractional Gaussian motion with the power spectrum such as $\mathrm{f}^{-\alpha}$, where the spectral index $0 \leq \alpha \leq 1$, and consider several choices of $\alpha$. In Figure 4, we measure the effective $Q$ factor for several values of $\alpha$ as a function of $\delta$. Smaller values of $\alpha$ (which correspond to slower decay of the spectrum) result in lesser sensitivity of the effective $Q$ factor to $\delta$. In the white noise spectrum $(\alpha=0)$, the effect is absent. The effect increases with an increase in the spectrum index. The deterioration of resonant properties of the resonator may be quite substantial; for example, the occurrence of background fluctuations with $\delta=0.2$ and $\alpha=1.0$ decreases the effective $Q$ factor more than 3 times, from $\sim 50$ to $\sim 15$.

Apart from the decrease in height and increase in width of the resonant peak, a secondary effect of the background fluctuations is a shift to lower frequency in the location of the maximum response. Figure 5 shows an expanded selection of $\delta$ values: we can see that the maximum response shifts to smaller frequencies $\omega<\Omega$ as $\delta$ increases.

\section{DISCUSSION}

The dayside magnetopause with a step-like change in magnetic field strength and plasma density may be imagined as a stressed membrane with reflecting boundaries in the northern and southern ionosphere, which can be resonantly excited by magnetosheath turbulence [Nenovski et al., 2007]. The MHD modeling to study the magnetospheric response to impulsive solar wind dynamic pressure increases has showed that waves with the $1.8 \mathrm{mHz}$ frequency are excited whose global

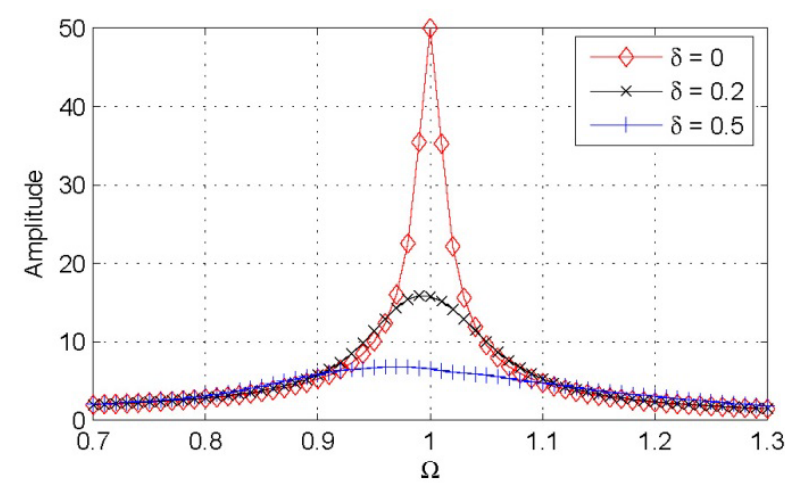

Figure 3. Amplitude of oscillations if $A=1, \omega=1, \gamma=0.01$, and eigenfrequency fluctuations with power spectrum $1 / f$. The height of the peak for each curve is the effective $Q$-factor 


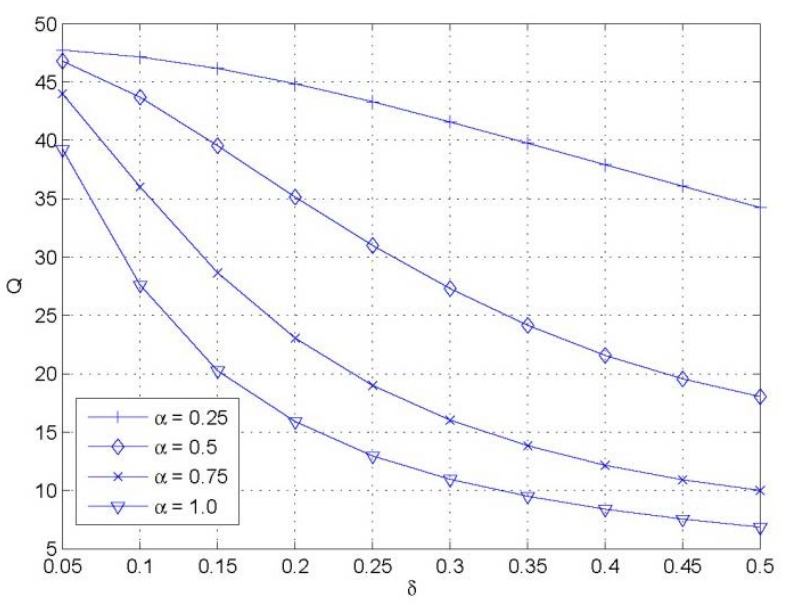

Figure 4. Maximum height (the Q-factor) of the resonant peak if $A=1, \omega=1, \gamma=0.01$, and eigenfrequency fluctuations with power spectrum $f^{-\alpha}$ for various $\delta$ and spectral slope $\alpha$

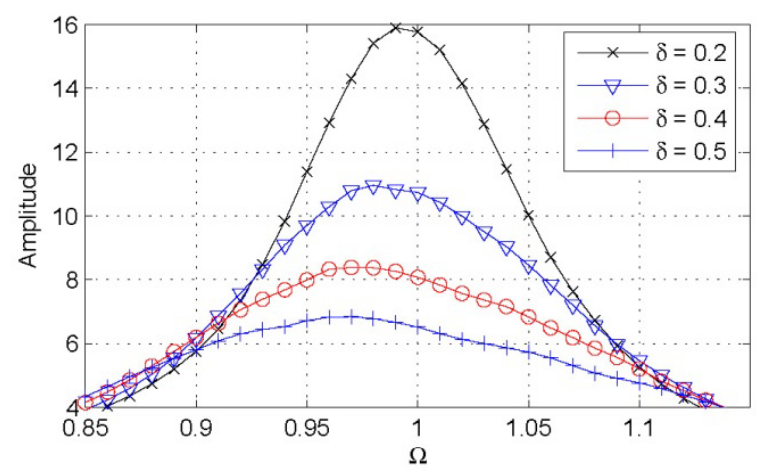

Figure 5. The frequency dependence of the maximum height ( $Q$ factor) of the resonant peak for expanded selection of $\delta$ values

properties cannot be explained by other known ULF wave modes [Hartinger et al., 2015]. These simulation results indicate that surface modes can be sustained in realistic magnetic field geometries with nonzero flow shear and finite current layer thickness at the magnetopause. Thus, magnetopause surface eigenmodes are a potential source of magnetospheric dayside ULF waves with frequencies less than $2 \mathrm{mHz}$. The energy of discrete spectrum mode (such as surface mode) is irreversibly converted into the energy of Alfvén continuum, so the surface mode can exist for a limited time. Therefore, only short-lived transient Pc6 oscillations can be associated with possible magnetopause modes, whereas longlasting quasi-periodic oscillations in the Pc5-6 band are probably related to the resonant response of magnetospheric shells at lower latitudes.

The surface mode on the dawn and dusk flanks of the magnetopause can be excited by the $\mathrm{KH}$ instability of the magnetosheath plasma flow around the magnetosphere. However, this mechanism is hardly operative in the near-noon sector, where the velocity of the plasma flow is low. A driver of magnetopause surface modes can be quasi-periodic fluctuations of the solar wind dynamic pressure, similar to a direct solar wind driving found for $\sim 1 / 3$ of Pc5 events [Viall et al., 2009]. Another possible driver can be fluctuations of the field-aligned current system at the boundary layer [Menk et al., 1992].
Typically, magnetospheric field line oscillations have been modeled in plasma with regular parameters. However, in a realistic plasma medium the eigenfrequency of the field line resonator $\Omega$ should experience inherited fluctuations because of stochastic variations in the geomagnetic field magnitude, field line length, and plasma density. We have considered the effect of the background homogeneous fluctuations on driven field line standing oscillations. In general, our estimates indicate the necessity to seriously consider the possible effective damping of ULF waves due to background magnetospheric turbulence. The effect considered here may help to explain a lack of narrowband ULF waves during magnetically disturbed periods, when the magnetospheric turbulence level is substantially elevated.

More specifically, this effect may be responsible for suppression of field line standing oscillations at the magnetopause. Deeper in the magnetosphere, away from the magnetopause, the magnetospheric magnetic field and plasma become more regular and the level of their fluctuations decreases. As a result, the resonant response to an external driving becomes evident in ground magnetometer data. The proposed mechanism can interpret the puzzling lack of ground response to surface modes or oscillations of the last field line near the OCB ionospheric projection; it merits further validation and verification.

The suppression of resonant field line oscillations depends not only on the level of fluctuations, but on their spectral form as well. More definitive conclusions require more detailed information about power and spectra of the background magnetospheric turbulence. The physics of the resonator in a stochastic medium is much richer than has been considered here. In particular, an increase in the fluctuation level can result in parametric excitation of the resonator without any external energy pumping. However, this and many other intriguing possibilities are beyond the scope of this paper.

The "plasma box" model considered is evidently oversimplified. In realistic geometry, field lines from the dayside near-magnetopause regions are squeezed into a narrow tube in the cusp region. This steep change of magnetic field line geometry can result in a partial reflection of Alfvén waves propagating toward the ionosphere along a field line [Pilipenko et al., 2005]. As a result, the standing mode with a higher frequency may appear not between conjugate ionospheres, but in a quasi-resonator along a field line part between reflection points. Finite-pressure effects can also impede fieldaligned propagation of Alfvén waves [Mager et al., 2009]. Hence, a part of the wave energy reaching the ionosphere would be substantially attenuated.

\section{CONCLUSION}

The multi-instrument studies of the latitudinal structure of specific dayside broadband Pc5-6 pulsations recorded by magnetometers covering near-cusp latitudes have shown that the maximum of the pulsation power occurs $1-2^{\circ}$ deeper in the magnetosphere than OCB determined either from the SuperDARN radar data or with a scanning photometer. To interpret the puzzling lack of the ground response to the last field line oscillations or surface mode at magnetopause, we suggest that 
stochastic fluctuations of the magnetospheric plasma and background magnetic field can suppress the excitation of standing MHD oscillations in a close vicinity of the magnetopause. To quantify this hypothesis, we have considered the model of a driven field line resonator with stochastic eigenfrequency fluctuations. This problem is studied analytically for a low level of fluctuations. The results of analytical calculations have proved the deterioration of resonant properties of the resonator owing to background fluctuations. The suppression of resonant field line oscillations depends not only on the level of fluctuations, but on their spectral form as well. This effect may explain the lack of ground response to magnetopause surface modes or oscillations of the last field line in the dayside magnetosphere. On the other hand, keeping in mind the small latitudinal shift, the location of the Pc6 latitudinal maximum can be used as a simple ground indicator, though biased, of the dayside OCB.

This work was supported by grant No. 246725 from the POLARPROG program of the Norwegian Research Council (VP, LB, DL, OK) and grant No. 16-35-00095 from the Russian Foundation for Basic Research (VB). We appreciate useful comments of both reviewers.

\section{REFERENCES}

Archer M.O., Hartinger M.D., Horbury T.S. Magnetospheric "magic" frequencies as magnetopause surface eigenmodes. Geophys. Res. Lett. 2013, vol. 40, pp. 5003-5008.

Archer M.O., Plaschke F. What frequencies of standing surface waves can the subsolar magnetopause support? J. Geophys. Res. 2015, vol. 120, pp. 3632-3646.

Baker K.B., Dudeney J.R., Greenwald R.A., Pinnock M., Newell P.T., Rodger A.S., Mattin N., Meng C.-I. HF radar signatures of the cusp and low-latitude boundary layer. J. Geophys. Res. 1995, vol. 100, pp. 7671-7695.

Bolshakova O.V., Kleimenova N.G., Kurazhkovskaya N.A. Polar cap dynamics using the observations of long period geo-magnetic pulsations. Geomagnetism and Aeronomy. 1988, vol. 28, pp. 661-665.

Borovsky J.E., Elphic R.C., Funsten H.O., Thomsen M.F. The Earth's plasma sheet as a laboratory for turbulence in high- $\beta$ MHD. J. Plasma Phys. 1997, vol. 57, pp. 1-34.

Chen L., Hasegawa A. A theory of long-period magnetic pulsations: 2. Impulse excitation of surface eigenmode. J. Geophys. Res. 1974, vol. 79, pp. 1033-1037.

Chen X.-C., Lorentzen D.A., Moen J.I., Oksavik K., Baddeley L.J. Simultaneous ground-based optical and HF radar observations of the ionospheric footprint of the open/closed field line boundary along the geomagnetic meridian. J. Geophys. Res. 2015, vol. 120, pp. 9859-9874.

Coult N., Pilipenko V., Engebretson M. Suppression of resonant field line oscillations by a turbulent background. Planet. Space Sci. 2007, vol. 55, pp. 694-700.

Dimentberg M.F. Nonlinear stochastic problems of mechanical oscillations. Moscow, Nauka Publ, 1980, 368 p. (In Russian).

Engebretson M.J., Hughes W.J., Alford J.L., Zesta E., Cahill L.J., Arnoldy R.L., Reeves G.D. Magnetometer array for cusp and cleft studies observations of the spatial extent of broadband ULF magnetic pulsations at cusp/cleft latitudes. J. Geophys. Res. 1995, vol. 100, pp. 19371-19386.

Fedorov E., Pilipenko V., Engebretson M.J. ULF wave damping in the auroral acceleration region. J. Geophys. Res. 2001, vol. 106, pp. 6203-6212.
Francia P., Lanzerotti L.J., Villante U., Lepidi S., Di Memmo D. A statistical analysis of low-frequency magnetic pulsations at cusp and cap latitudes in Antarctica. J. Geophys. Res. 2005, vol. 110, A02205. DOI: 10.1029/2004JA010680.

Gardiner C.W. Handbook of Stochastic Methods. 3rd ed. Springer-Verlag, 2004, 409 p.

Goossens M., Ruderman M.S., Hollweg J.V. Dissipative MHD solutions for resonant Alfven waves in 1D magnetic flux tubes. Solar Phys. 1995, vol. 157, pp. 75-102.

Hartinger M.D., Plaschke F., Archer M.O., Welling D.T., Moldwin M.B., Ridley A. The global structure and time evolution of dayside magnetopause surface eigenmodes. Geophys. Res. Lett. 2015, vol. 42, pp. 2594-2602.

Hollweg J.V. Resonant absorption of magnetohydrodynamic surface waves: viscous effects. Astron. J. 1987, vol. 320, pp. 875-883.

Hollweg J.V. A simple mechanical model for resonance absorption: The Alfven resonance. J. Geophys. Res. 1997, vol. 102, pp. 24127-24137.

Johnsen M.G., Lorentzen D.A. The dayside open/closed field line boundary as seen from space and ground-based instrumentation. J. Geophys. Res. 2012, vol. 117, A03320. DOI: 10.1029/2011JA016983.

Kozyreva O.V., Pilipenko V.A., Engebretson M.J., Klimushkin D.Yu., Mager P.N., Correspondence between the ULF wave power distribution and auroral oval. Solar-Terr. Phys. 2016, vol. 2, pp. 46-65. DOI: 10.12737/16848

Lanzerotti L.J., Shono A., Fukunishi H., Maclennan C.G. Long-period hydromagnetic waves at very high geomagnetic latitudes. J. Geophys. Res. 1999, vol. 104, pp. 28423-28435.

Leonovich A.S., Mazur V.A. Resonance excitation of standing Alfvén waves in an axisymmetric magnetosphere (Monochromatic oscillations). Planet. Space Sci. 1989, vol. 37, pp. 1095-1108.

Lorentzen D.A., Deehr C.S., Minow J.I., Smith R.W., Stenbaek-Neielsen H.C., Sigernes F., Arnoldy R.L., Lynch K. SCIFER-Dayside auroral signatures of magnetospheric energetic electrons. Geophys. Res. Lett. 1996, vol. 23, pp. 1885-1888.

Lorentzen D.A., Moen J., Auroral proton and electron signatures in the dayside aurora. J. Geophys. Res. 2000, vol. 105, pp. 12733-12745.

Lysak R.L., Dum C.T. Dynamics of magnetosphereionosphere coupling including turbulent transport. J. Geophys. Res. 1983, vol. 88, pp. 365-380.

Mager P.N., Klimushkin D.Yu., Pilipenko V.A., Schaefer S. Field-aligned structure of poloidal Alfvén waves in a finite pressure plasma. Ann. Geophysicae. 2009, vol. 27, pp. 38753882.

Mazur V.A., Chuiko D.A. Kelvin-Helmholtz instability on the magnetopause, magnetohydrodynamic waveguide in the outer magnetosphere, and Alfvén resonance deep in the magnetosphere. Plasma Phys. Rep. 2013, vol. 39, pp. 488-503.

McHarg M.G., Olson J.V., Newell P.T. ULF cusp pulsations: diurnal variations and interplanetary magnetic field correlations with ground-based observations. J. Geophys. Res. 1995, vol. 100, pp. 19729-19742.

Menk F.W., Hansen H.J., Dunlop I.S., Fraser B.J., Newell P.T., Meng C.I., Morris R.J. ULF wave sources at polar cusp and boundary layer latitudes. Solar-Terrestrial Energy Program. The initial results from STEP facilities and theory campaigns. Pergamon Press, 1992, pp. 301-312.

Milan S.E., Lester M., Cowley S.W.H., Moen J., Sandholt P.E., Owen C.J. Meridian-scanning photometer, coherent HF radar, and magnetometer observations of the cusp: a case study. Ann. Geophysicae. 1999, vol. 17, pp. 159-172.

Moen J., Carlson H.C., Milan S.E., Shumilov N., Lybekk B., Sandholt P.E., Lester M. On the collocation between dayside auroral activity and coherent HF radar backscatter. Ann. Geophysicae. 2001, vol. 8, pp. 1531-1549. 
Nenovski P., Villante U., Francia P., Vellante M., Bochev A. Do we need a surface wave approach to the magnetospheric resonances? Planetary and Space Sci. 2007, vol. 55, pp. 680-693.

Newell P.T., Meng C.-I. The cusp and the cleft/boundary layer: low-altitude identifications and statistical local time variation. J. Geophys. Res. 1988, vol. 93, pp. 14549-14556.

Newton R.S., Southwood D.J., Hughes W.J. Damping of geomagnetic pulsations by the ionosphere. Planet. Space Sci. 1978, vol. 26, pp. 201-209.

Oksavik K., Søraas F., Moen J., Burke W.J. Optical and particle signatures of magnetospheric boundary layers near magnetic noon: Satellite and ground-based observations. J. Geophys. Res. 2000, vol. 105, pp. 27555-27568.

Pilipenko V., Belakhovsky V., Engebretson M.J., Kozlovsky A., Yeoman T. Are dayside long-period pulsations related to the cusp? Ann. Geophysicae. 2015, vol. 33, pp. 395-404.

Pilipenko V., Mazur N., Fedorov E., Engebretson M.J., Murr D.L. Alfven wave reflection in a curvilinear magnetic field and formation of Alfvenic resonators on open field lines. J. Geophys. Res. 2005, vol. 110, A10S05. DOI: 10.1029/ 2004JA010755.

Plaschke F., Glassmeier K.-H., Constantinescu O.D., Mann I. R., Milling D.K., Motschmann U., Rae I.J. Statistical analysis of ground based magnetic field measurements with the field line resonance detector. Ann. Geophysicae. 2008, vol. 26, pp. 3477-3489.

Plaschke F., Glassmeier K.H. Properties of standing Kruskal-Schwarzschild modes at the magnetopause. Ann. Geophysicae. 2011, vol. 29, pp. 1793-1807.

Plaschke F., Glassmeier K.-H., Auster H.U., Constantinescu O.D., Magnes W., Angelopoulos V., Sibeck D.G., McFadden J.P. Standing Alfven waves at the magnetopause. Geophys. Res. Lett. 2009a, vol. 36, L02104. DOI: 10.1029/ 2008 GL036411.

Plaschke F., Glassmeier K.-H., Sibeck D.G., Auster H.U., Constantinescu O.D., Angelopoulos V., Magnes W. Magnetopause surface oscillation frequencies at different solar wind conditions. Ann. Geophysicae. 2009b, vol. 27, pp. 4521-4532.

Rankin R., Samson J.C., Tikhonchuk V.T. Discrete auroral arcs and nonlinear dispersive field line resonances. Geophys. Res. Lett. 1999, vol. 26, pp. 663-666.

Rodger A.S., Mende S.B., Rosenberg T.J., Baker K.B., Simultaneous optical and HF radar observations of the ionospheric cusp. Geophys. Res Lett. 1995, vol. 22, pp. 2045-2048.

Urban K.D., Gerrard A.J., Bhattacharya Y., Ridley A.J., Lanzerotti L.J., Weatherwax A.T. Quiet time observations of the open-closed boundary prior to the CIR-induced storm of 9 August 2008. Space Weather. 2011, vol. 9, S11001.

Viall N.M., Kepko L., Spence H.E. Relative occurrence rates and connection of discrete frequency oscillations in the solar wind density and dayside magnetosphere. J. Geophys. Res. 2009, vol. 114, A01201. DOI: 10.1029/2008JA013334.

Villante U., Lepidi S., Francia P., Meloni A., Palangio P. Long period geomagnetic field fluctuations at Terra Nova Bay Antarctica. Geophys. Res. Lett. 1997, vol. 24. pp. 1443-1446.

Yeoman T.K., Lester M., Cowley S.W.H., Milan S.E., Moen J., Sandholt P.E. Simultaneous observations of the cusp in optical, DMSP and HF radar data. Geophys. Res. Lett. 1997, vol. 24, pp. 2251-2254.

Yumoto K., Pilipenko V., Fedorov E., Kurneva N., Shiokawa K. The mechanisms of damping of geomagnetic pulsations. J. Geomag. Geoelectr. 1995, vol. 47, pp. 163-176.

\section{APPENDIX}

The results that can be obtained with the successive approximation method are restricted by the condition of small $\delta$. In subsequent approximations $(i=1,2, \ldots)$, the fluctuation-induced correction to the solution of (1) is determined by a recurrence formula, which follows from the infinite system of equations

$$
\begin{aligned}
& x_{i}(t)=-\frac{\Omega^{2}}{\omega_{1}^{2}} \times \\
& \times \int_{-\infty}^{t} \exp \left[-\gamma\left(t-t^{\prime}\right)\right] \sin \left[\omega_{1}\left(t-t^{\prime}\right)\right] x_{i-1}\left(t^{\prime}\right) \xi\left(t^{\prime}\right) d t^{\prime} .
\end{aligned}
$$

The time-averaged behavior of the oscillatory system is $\langle x(t)\rangle=x_{0}(t)+\delta^{2}\left\langle x_{2}(t)\right\rangle$, as the linear response to fluctuations vanishes, that is $\left\langle x_{1}(t)\right\rangle=0$. Using (A1), we can obtain the second order correction

$$
\begin{aligned}
& \frac{\left\langle x_{2}(t)\right\rangle}{b_{0}}=\frac{\Omega^{4}}{\omega_{1}^{2}} \int_{-\infty}^{t} \mathrm{e}^{-\gamma\left(t-t^{\prime}\right)} \sin \left[\omega_{1}\left(t-t^{\prime}\right)\right] d t^{\prime} \times \\
& \times \int_{-\infty}^{t^{\prime}} K\left(t^{\prime}-t^{\prime \prime}\right) \mathrm{e}^{-\gamma\left(t^{\prime}-t^{\prime \prime}\right)} \sin \left[\omega_{1}\left(t^{\prime}-t^{\prime \prime}\right)\right] \sin \left(\omega t^{\prime \prime}+\varphi_{0}\right) d t^{\prime \prime},
\end{aligned}
$$

where $K(\tau)$ is the auto-correlation function. After integrating and neglecting the terms $\sim \delta^{4}$, the relationships (A1) and (A2) can be combined into (5). Here we introduce the functions

$$
\begin{aligned}
& J^{( \pm)}=\int_{0}^{\infty} K(\tau) \exp (-\gamma \tau) \cos \left[\left(\omega \pm \omega_{1}\right) \tau\right] d \tau, \\
& I^{( \pm)}=\int_{0}^{\infty} K(\tau) \exp (-\gamma \tau) \sin \left[\left(\omega \pm \omega_{1}\right) \tau\right] d \tau .
\end{aligned}
$$

The relationships (A2, A3) enable us to examinn how the amplitude of the resonant oscillations sh change under the influence of stochastic fluctuation eigenfrequency. Formula (5) shows that when the spectral density $\Phi(\omega)$ of $\xi(t)$ fluctuations is a non-growing function, these fluctuations cause a decrease in the average amplitude of resonant oscillations. Indeed, using the Wiener-Khinchin theorem, Dimentberg [1980] proved strictly that if the spectral density of fluctuations $\Phi(\omega)$ decreases with frequency, i.e. $d_{\omega} \Phi \leq 0$ for all $\omega \geq 0$, then the term in square brackets in $(5,6)$ is always positive and $b / b_{0}^{(\max )}<1$. Thus, the occurrence of background turbulence $\delta \neq 0$ causes a decrease and widening of the resonant amplitude-frequency response of the system.

How to cite this article

Pilipenko V.A., Kozyreva O.V., Baddeley L., Lorentzen D., Belakhovsky V.B. Suppression of the dayside magnetopause surface modes. Solar-Terrestrial Physics. 2017. Vol. 3, No. 4. P. 17-25. DOI: 10.12737/stp-34201702 